\title{
SELECTED ASPECTS OF DESIGNING DEEP EXCAVATIONS
}

\author{
RAFAE F. OBRZUD \\ PhD Eng. Geotechnical computer consultant, Karakas \& Français, Switzerland, \\ e-mail: rafal.obrzud@gmail.com
}

SÉBASTIEN HARTMANN

MSc candidate, École Polytechnique Fédérale de Lausanne, Switzerland

KRZYSZTOF PODLEŚ

PhD Eng. Cracow University of Technology

\begin{abstract}
This paper analyzes two approaches to serviceability limit state (SLS) verification for the deep excavation boundary value problem. The verification is carried out by means of the finite element (FE) method with the aid of the commercial program ZSoil v2014. In numerical simulations, deep excavation in non-cohesive soil is supported with a diaphragm wall. In the first approach, the diaphragm wall is modeled with the Hookean material assuming reduced average stiffness and possible concrete cracking. The second approach is divided into two stages. In the first stage, the wall is modeled by defining its stiffness with the highest nominal Young's modulus. The modulus makes it possible to find design bending moments which are used to compute the minimal design cross-section reinforcement for the retaining structure. The computed reinforcement is then used in a non-linear structural analysis which is viewed as the "actual" SLS verification.

In the second part, the paper examines the same boundary value problem assuming that the excavation takes place in quasiimpermeable cohesive soils, which are modeled with the Hardening Soil model. This example demonstrates the consequences of applying the steady-state type analysis for an intrinsically time-dependent problem. The results of this analysis are compared to the results from the consolidation-type analysis, which are considered as a reference. For both analysis types, the two-phase formulation for partially-saturated medium, after Aubry and Ozanam, is used to describe the interaction between the soil skeleton and pore water pressure.
\end{abstract}

Key words: deep excavation, serviceability limit state, steady-state analysis, consolidation analysis, undrained behavior analysis, ZSoil PC

\section{INTRODUCTION}

Deep excavations in urban areas generally result in ground movements that can induce significant damage to adjacent buildings and services. In order to avoid such movements or reduce their impact, the excavations are supported by stiff diaphragm walls, struts and anchors, which are installed as the excavation progresses. However, changes in the vertical stress relief associated with an excavation cause soil deformations and associated wall deflections. This induces surface settlements - even if the retaining walls are prevented from moving horizontally - and deep-seated inward displacements of the walls that cannot be controlled by the struts or anchors that are installed within the excavation itself (Burland et al. [6]). A rigorous soil-structure interaction analysis should therefore include an adequate choice of constitutive laws describing both soil and structural elements, a reliable selection of parameters, as well as the adoption of the correct type of analysis (i.e., time-dependent effects). These elements make the analysis of static soilstructure interaction problems - particularly with reference to serviceability limit states - one of the most challenging tasks in modern geotechnical engineering.

The goal of this paper is to examine two approaches to SLS verification for the deep excavation boundary value problem. The verification is carried out by means of the finite element (FE) method with the aid of the commercial program ZSoil v2014, using a case study provided in the program. Advanced nu- 
merical modeling involves description of soil behavior with the aid of the Hardening Soil (HS) model, see Section 2.3. In numerical simulations, deep excavation in non-cohesive soil, known as excavation in Berlin Sand (Schweiger [14]), is supported with an anchored diaphragm wall. In the first approach, the diaphragm wall is modeled with the Hookean material assuming reduced average stiffness due to possible concrete cracking.

The second approach is divided into two stages. In the first stage, the wall is modeled with the highest nominal Young's modulus. This modulus makes it possible to find design bending moments which are used to compute the minimal design cross-section reinforcement for the retaining structure. The computed reinforcement is then used in a non-linear structural analysis which is viewed as the "actual" SLS verification.

In the second part of the paper, the same boundary value problem is re-analyzed assuming that the excavation takes place in quasi-impermeable cohesive soils, described with the Hardening Soil model. The soil is considered as normally consolidated. This example demonstrates and discusses consequences of applying the steady-state analysis type for intrinsically time-dependent problem. In this case, the effect of suction due to partially-saturated soil is discussed. The results of this analysis are compared to the results from the consolidation-type analysis, which are considered as a reference. For both analysis types, the two-phase formulation for partially saturated medium, after Aubry and Ozanam (Aubry and Ozanam [4]), is briefly recalled in Section 3.1, and used to describe the interaction between the soil skeleton and pore water pressure.

\section{SERVICEABILITY LIMIT STATE CONTROL AND WALL DESIGN}

This section examines two approaches to SLS verification for the deep excavation boundary value problem. By definition, serviceability refers to conditions under which an engineering system is considered useful. In the context of deep excavation, serviceability refers to conditions other than the strength of soil or structural members that render the system performable and stable. SLS design of deep excavation includes factors such as overall stability of soil and the retaining structure, deflection and cracking of the diaphragm wall and, finally, settlements of existing adjacent buildings.
The two approaches considered are described below and their work flows are schematically presented in Fig. 1.

Method A consists in verification of the effectiveness of a retaining structure by means of a single simulation. The verification is meant to provide the magnitude of wall deflections and soil deformations. Such simulations are often carried out before the dimensioning of the concrete wall members, meaning that the rebar reinforcement is unknown. Therefore, in order to istically approximate soil-structure interactions, the retaining wall is modeled with the elastic model, assuming a reduced Young's modulus. This modulus accounts for concrete cracking due to bending-induced tension ${ }^{1}$. The characteristic concrete stiffness of about $E_{c}=34 \mathrm{GPa}$, which is given by EC2 [3], can be intuitively reduced to $E_{c}=20 \mathrm{GPa}$ (experience shows that this value is commonly applied in geotechnical practice in order to account for concrete cracking). In this case, the global stiffness reduction applies to all wall members meaning that we assume that all wall members undergo cracking. The reduced flexional rigidity of the wall allows deformations to be mobilized in soil behind the retaining structure, resulting in an overall good approximation of displacement fields.

However, experience shows that the results obtained with such a simplified model adopted to SLS analysis, are used to retrieve the internal forces that are developed in the wall members. As a result, bending moment, normal and shear force (MNV) envelopes risk being carelessly used to design reinforcing rebars. It is clear that a less rigid structure can provide an underestimated magnitude of the characteristic bending moment $M_{k}$, with regard to the structural safety that is demanded by the national codes. The design value of the bending moment $M_{d}$ is computed by applying the partial factor $\gamma_{f}$, which can be considered as equal to 1.35 (EC0 [2])

$$
M_{d}=\gamma_{f} \times M_{k}=1.35 \cdot M_{k} .
$$

Notice that in the case of the simplified approach, $M_{k}$ is obtained for reduced structure rigidity, $M_{k}^{A}=M_{k}\left(E_{c}=20 \mathrm{GPa}\right)$.

The second approach, here called Method B, considers performing SLS verification in two steps. The first step consists of running a FE simulation with the retaining wall obeying Hooke's law. In this case, mature concrete is defined using its initial stiff-

${ }^{1}$ Creep- and shrinkage-induced cracking are neglected in this paper. 


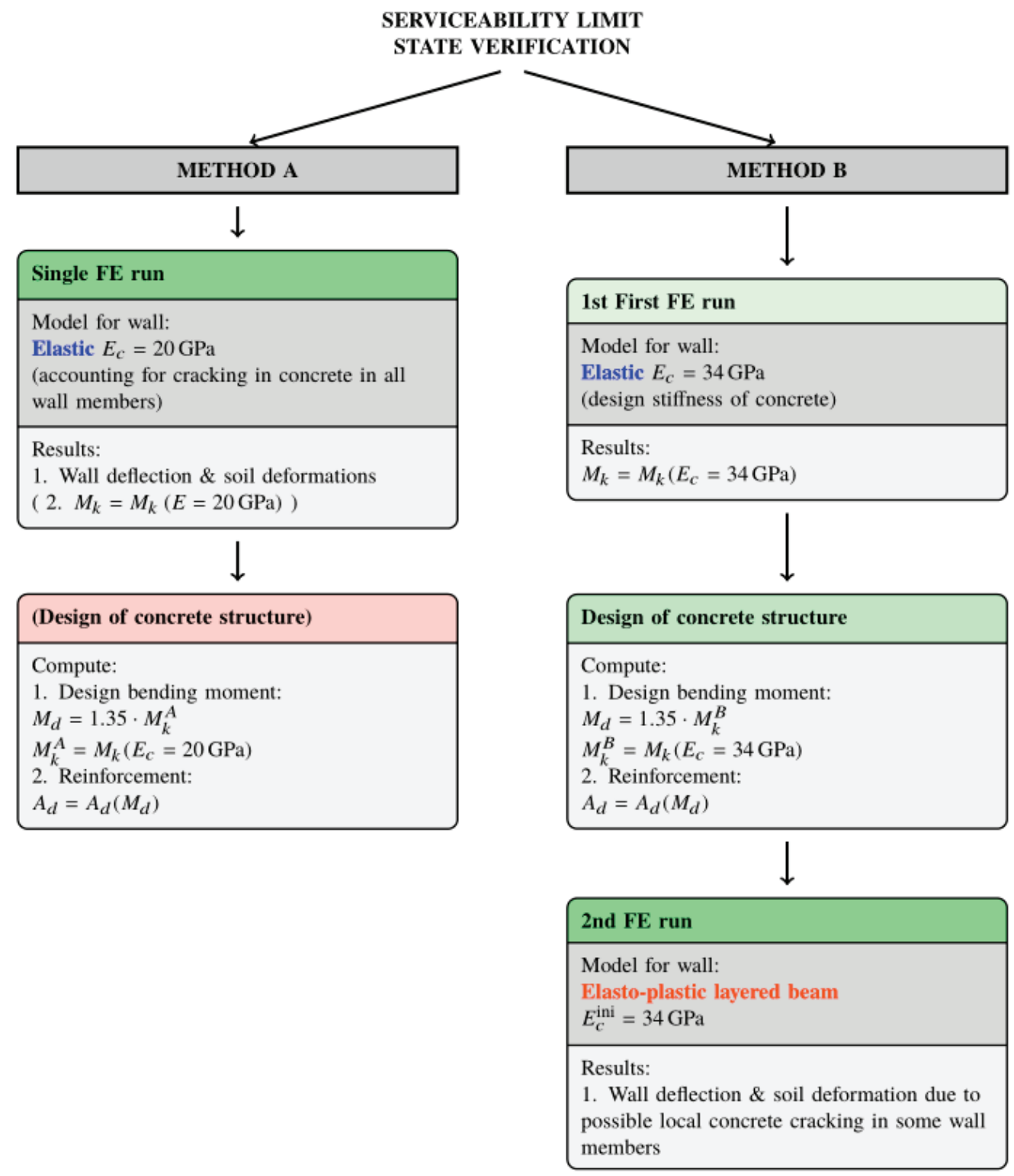

Fig. 1. Schematic workflows for two approaches for serviceability limit state check

ness $E_{c} \approx 34 \mathrm{GPa}$ (cf. EC0 [2]). The first step involves computing internal forces which are used for designing the reinforcement $A_{\mathrm{sd} \text {,min. }}$. In this paper, minimal cross-section reinforcement was computed using the rectangular stress distribution model, described in the Appendix.

The calculated reinforcement area can be used for the second FE run in which the retaining wall is modeled by means of the elasto-plastic beam elements, described in Section 2.1. The second simulation can be viewed as an "actual" SLS analysis because the retaining structure is also modeled as an elasto-plastic material. As a consequence, a local stiffness reduction may occur in members subjected to extensive bending. In reality, local stiffness reduction is due to progressive cracking of concrete section, which is caused, among other factors, by tensile stresses and strains. In the numerical model, the local stiffness reduction corresponds to progressive reduction of the secant stiffness in the layers which define the cross-section of concrete. The stiffness is reduced following the anisotropic uni-axial stress-strain curve for concrete, and occurs predominantly in the direction of tensile stress.

\subsection{ELASTO-PLASTIC MODEL FOR WALL}

In the case of the non-linear structural analysis (2nd FE run in Method B), the retaining wall is modeled by means of non-linear beam cross-sections which are defined using multi-layered beam elements 
(refer to ZSO [1]). In these elements, the core material section is divided into 20 concrete sub-layers. Each of these layers has been described by a non-linear stressstrain relation which can be found in EC2 [3] (Fig. 2)

$$
\frac{\sigma_{c}}{f_{c m}}=\frac{k \eta-\eta^{2}}{1+(k-2) \eta}
$$

where

$$
\begin{aligned}
& \eta=\varepsilon_{c} / \varepsilon_{c 1}, \\
& \varepsilon_{c 1} \text { is the strain at peak stress: } 0.7 f_{c m}^{0.31}, \\
& \varepsilon_{c u 1}=3.5 \text { for } f_{\mathrm{ck}}<50 \mathrm{MPa}, \\
& k=1.1 E_{c m} \times\left|\varepsilon_{c 1}\right| / f_{c m} .
\end{aligned}
$$

The characteristics used in the non-linear structural analysis for C30 class concrete are summarized in Table 1. The stress-strain relation for concrete, which has been adopted for the numerical simulation, is presented in Fig. 3.

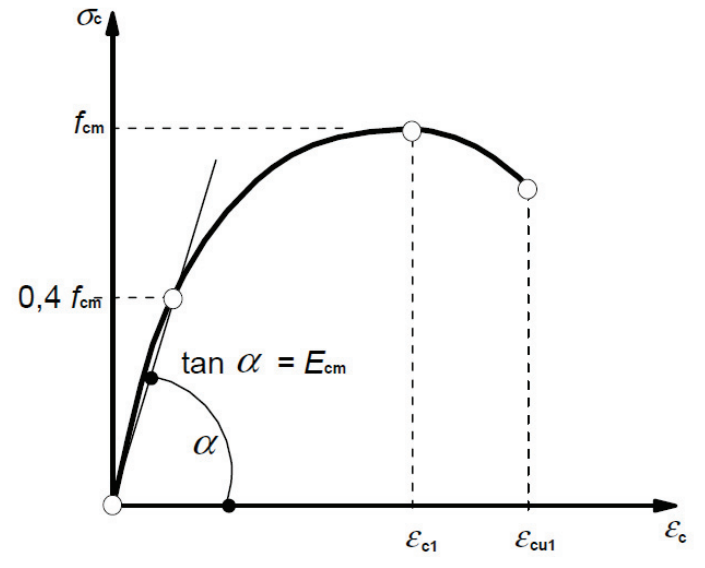

Fig. 2. Stress-strain relation for non-linear structural analysis (EC2 [3])

The strain-stress relation for the tensile branch in core material has been defined by the axial tensile strength of concrete $f_{c t k}$.
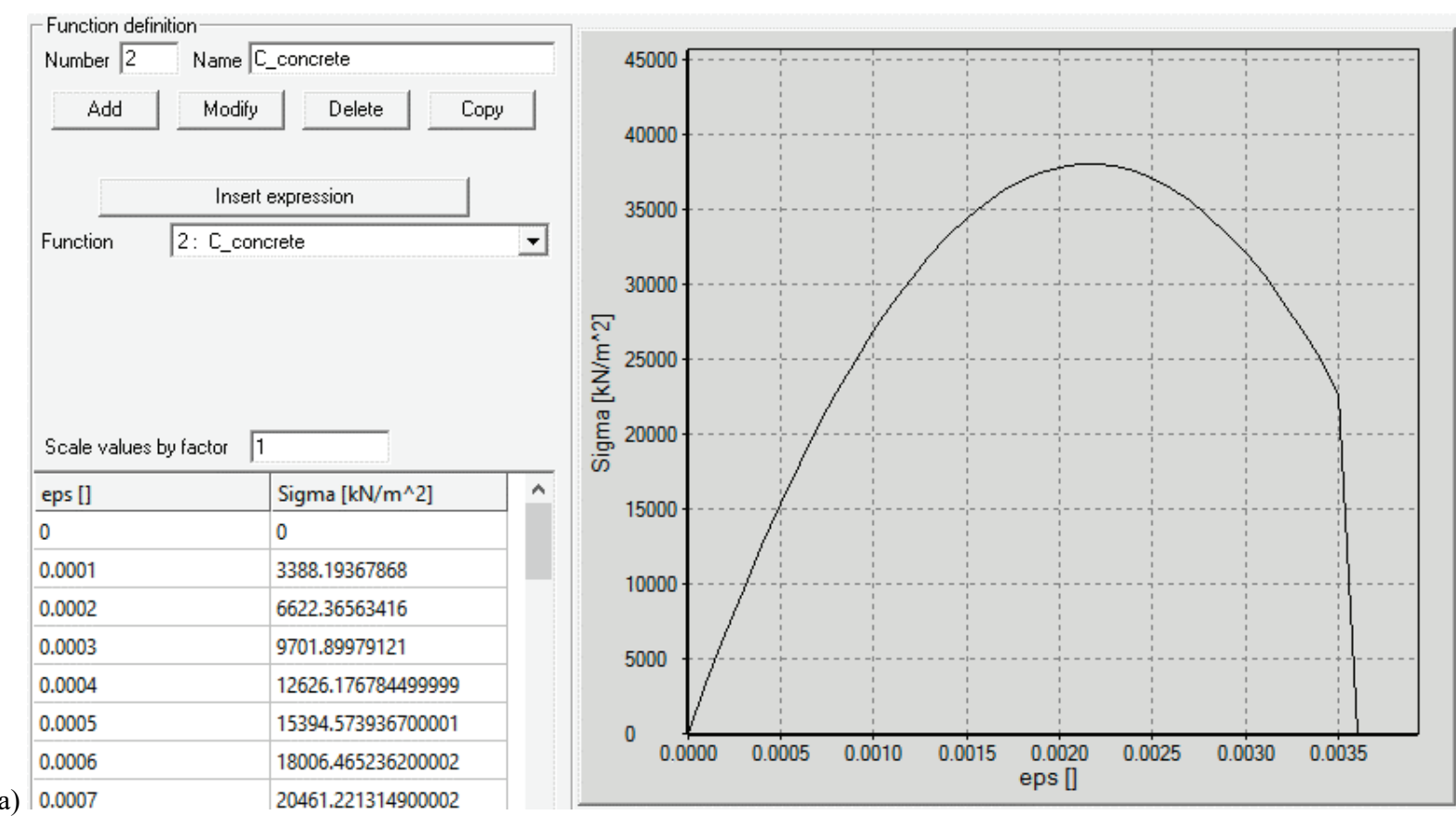

Additional layers
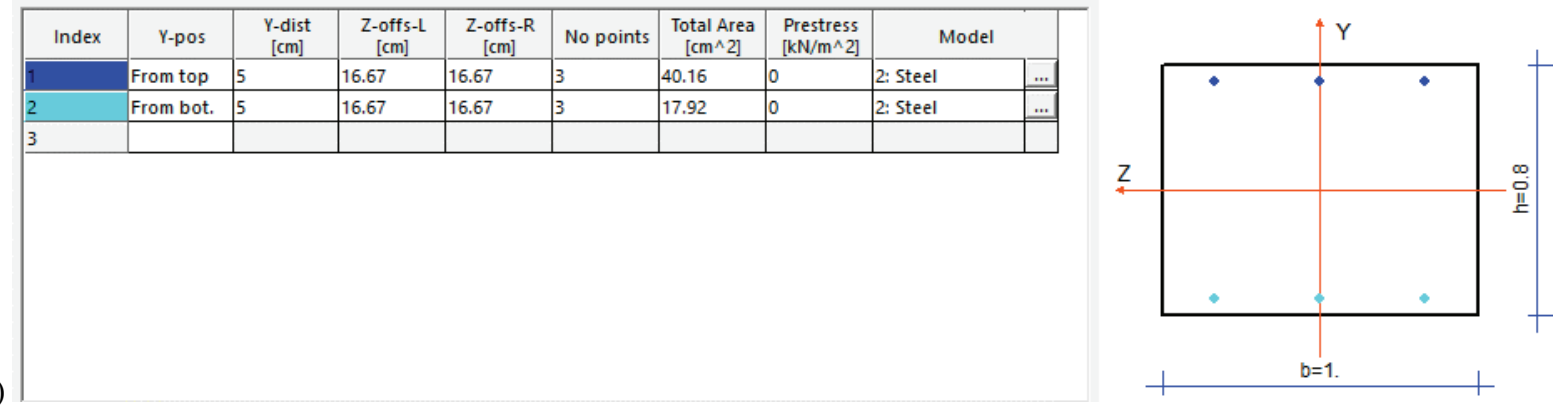

Fig. 3. Adopted stress-strain relation for non-linear structural analysis:

(a) adopted relationship for concrete $\mathrm{C} 30$, (b) assumed steel rebar reinforcement 
Table 1. Characteristics used in FE simulation for the non-linear model of retaining wall

\begin{tabular}{|c|c|c|c|c|}
\hline \multicolumn{5}{|c|}{ Concrete } \\
\hline $\begin{array}{l}\text { Initial } \\
\text { concrete } \\
\text { stiffness }\end{array}$ & $\begin{array}{l}\text { Poisson's } \\
\text { ratio }\end{array}$ & $\begin{array}{c}\text { Sectional } \\
\text { height }\end{array}$ & $\begin{array}{l}\text { Mean value } \\
\text { of concrete } \\
\text { cylinder } \\
\text { compressive } \\
\text { strength }\end{array}$ & $\begin{array}{l}\text { Characteristic } \\
\text { axial tensile } \\
\text { strength } \\
\text { of concrete }\end{array}$ \\
\hline$E_{c}^{\mathrm{ini}}[\mathrm{GPa}]$ & $v[-]$ & $h[\mathrm{~m}]$ & $f_{c m}[\mathrm{MPa}]$ & $f_{c t k}[\mathrm{MPa}]$ \\
\hline 34 & 0.2 & 0.8 & 38 & 0.1 \\
\hline \multicolumn{5}{|c|}{ Steel } \\
\hline $\begin{array}{l}\text { Young's } \\
\text { modulus }\end{array}$ & $\begin{array}{l}\text { Poisson's } \\
\text { ratio }\end{array}$ & $\begin{array}{l}\text { Sectional } \\
\text { area }\end{array}$ & $\begin{array}{l}\text { Characteristic } \\
\text { yield strength } \\
\text { of reinforcement }\end{array}$ & $\begin{array}{l}\text { Design yield } \\
\text { strength } \\
\text { of reinforcement }\end{array}$ \\
\hline$E_{s}[\mathrm{GPa}]$ & $v[-]$ & $A\left[\mathrm{~m}^{2}\right]$ & $f_{y k}[\mathrm{MPa}]$ & $f_{y d}[\mathrm{MPa}]$ \\
\hline 210 & 0.3 & 10.26 & 500 & 435 \\
\hline
\end{tabular}

Rebar reinforcement is represented by additional layers in the beam cross-section. The reinforcement is defined by its cross- sectional area $A_{\text {sd. }}$. The rebar sets can be positioned with respect to the cross-section as shown in Fig. 3b. The steel rebars in the simulations presented are represented by an elasto-plastic material for which characteristics are given in Table 1.

A simplified stress-strain relation for the design of a cross-section was used to compute the necessary reinforcement for members of the wall. The procedure is presented in the Appendix. This approach assumes that (i) the contribution of the compressed rebars is not considered, and (ii) tensile resistance of concrete is neglected $\left(f_{c t k}\right)$.

\subsection{PROBLEM STATEMENT}

A comparison of the two methods is carried out with the aid of the numerical model which has been analyzed for the case study of an excavation in sand soil in Berlin (refer to Obrzud and Truty [11] being the part of ZSoil manual).

The FE model, which represents the boundary value problem of $16.8 \mathrm{~m}$ excavation, is shown in Fig. 4. The excavation takes place in two sand layers and has been divided into four excavation sub-stages presented in Fig. 14. The interface between the wall and soil is governed by the Coulomb frictional model

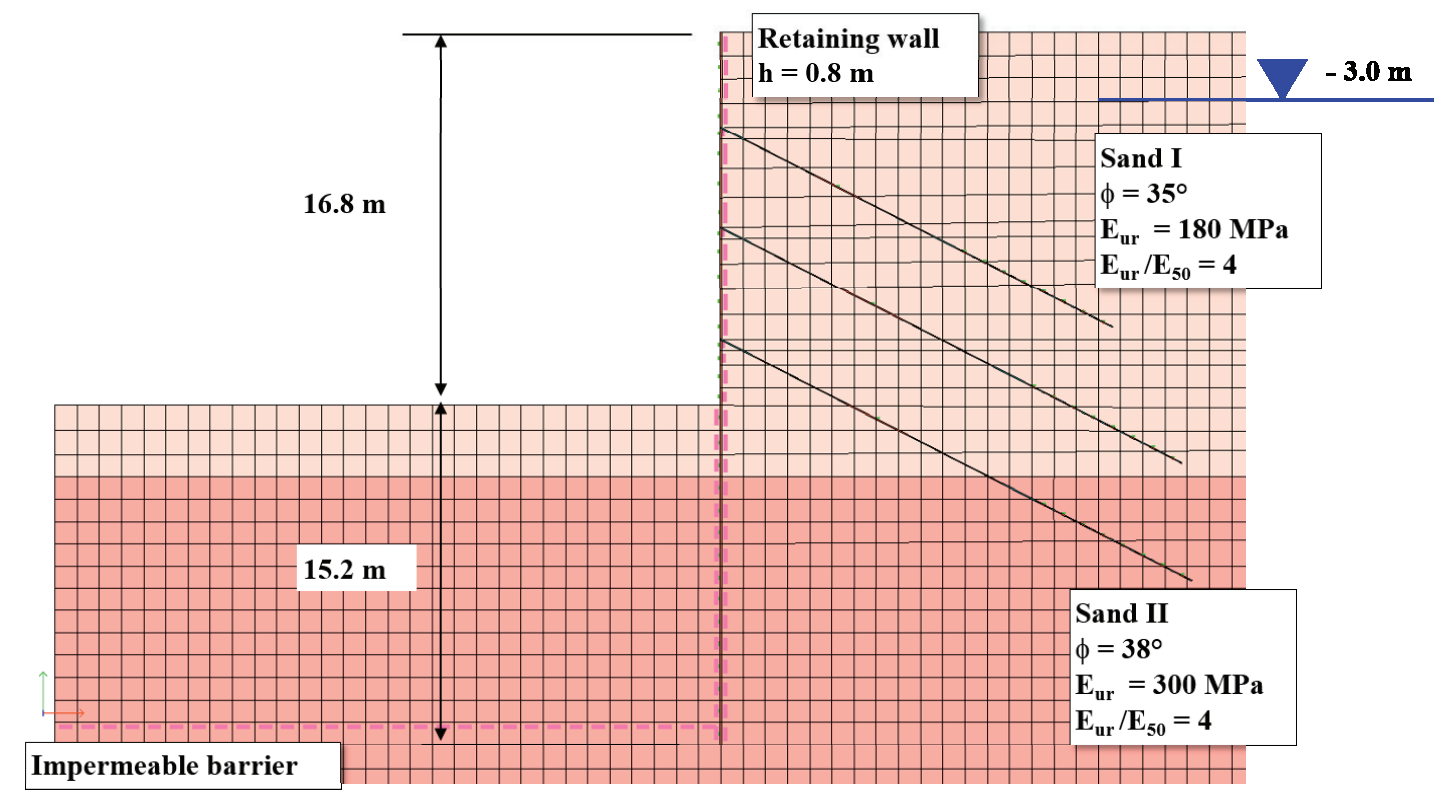

Fig. 4. Original problem statement for excavation in Berlin (Obrzud and Truty [11]) 
which is described by the effective parameters, friction angle $\phi^{\prime}=28^{\circ}$ and cohesion $c^{\prime}=0 \mathrm{kPa}$. A hydraulic barrier, which was installed to prevent water inflow during the excavation, has been modeled by introducing interface elements for which continuity applies only to the displacement variables, i.e., no fluid flow is allowed. The position of the ground water table (GWT), originally located at $-3.0 \mathrm{~m}$, was controlled by the fluid head pressure boundary conditions applied at the symmetry plane down to the impermeable barrier.

The presstressed anchors are introduced $0.5 \mathrm{~m}$ above the bottom of excavation stages in the following order:

- 1st row: $-2.30 \mathrm{~m}$, total legnth $19.8 \mathrm{~m}$, sealed zone $8.0 \mathrm{~m}$, prestressing force $\mathrm{P}_{0}=768 \mathrm{kN}$,

- 2nd row: $-9.30 \mathrm{~m}$, total legnth $23.3 \mathrm{~m}$, sealed zone $8.0 \mathrm{~m}$, prestressing force $\mathrm{P}_{0}=945 \mathrm{kN}$

- 3rd row: $-14.35 \mathrm{~m}$, total legnth $23.8 \mathrm{~m}$, sealed zone $8.0 \mathrm{~m}$, prestressing force $\mathrm{P}_{0}=980 \mathrm{kN}$.

\subsection{SOIL MODEL}

In this paper, the Hardening Soil (HS) model has been adopted to describe complex soil behavior (Schanz [12], Schanz et al. [13], Benz [5], ZSO [3], Obrzud and Truty [11]). This model has received a lot of attention of geotechnical engineers over the past fifteen years, as it is able to accurately predict the deformation and stress states for commonlysolved boundary problems, such as deep excavations, tunneling, shallow or deep foundations, and pile rafts.

The Hardening Soil model was designed by Schanz [12] and Schanz et al. [13] in order to reproduce basic macroscopic phenomena exhibited by soils, such as:

- densification - a decrease of voids volume in soil due to plastic deformations,

- stress dependent stiffness - observed phenomena of increasing stiffness moduli with increasing stress level,

- soil stress history - accounting for preconsolidation effects,

- plastic yielding - development of irreversible strains with reaching a yield criterion,

- dilatation - an occurrence of negative volumetric strains during shearing.

Contrary to other models such as the Cap model or the Modified Cam Clay, the magnitude of prefailure deformations can be modeled more accurately by incorporating three input stiffness charac- teristics corresponding to the triaxial loading moduli $\left(E_{50}\right)$, the triaxial unloading-reloading stiffness $\left(E_{\text {ur }}\right)$ (Fig. 5), and controlling the rate of volumetric plastic deformation based on a given tangent oedometric modulus $\left(E_{\text {oed }}\right)$.

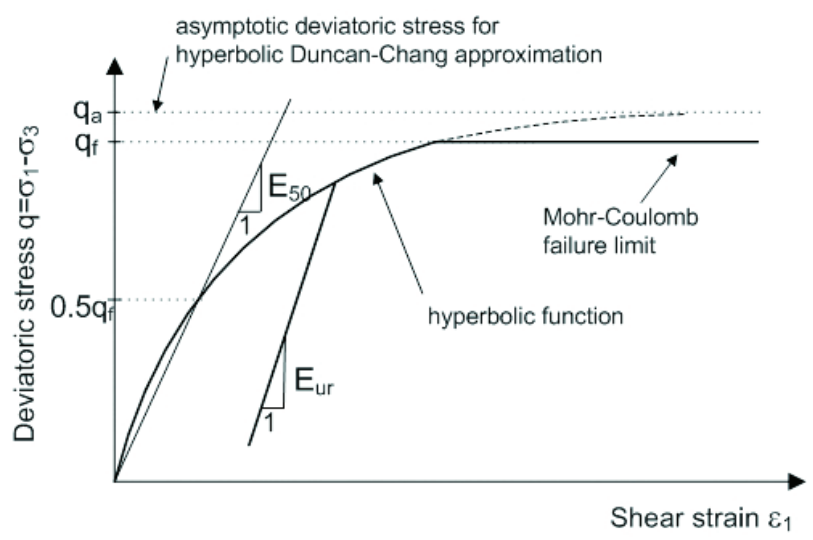

Fig. 5. Hyperbolic stress-strain relationship and the definition of different moduli in the triaxial drained test condition

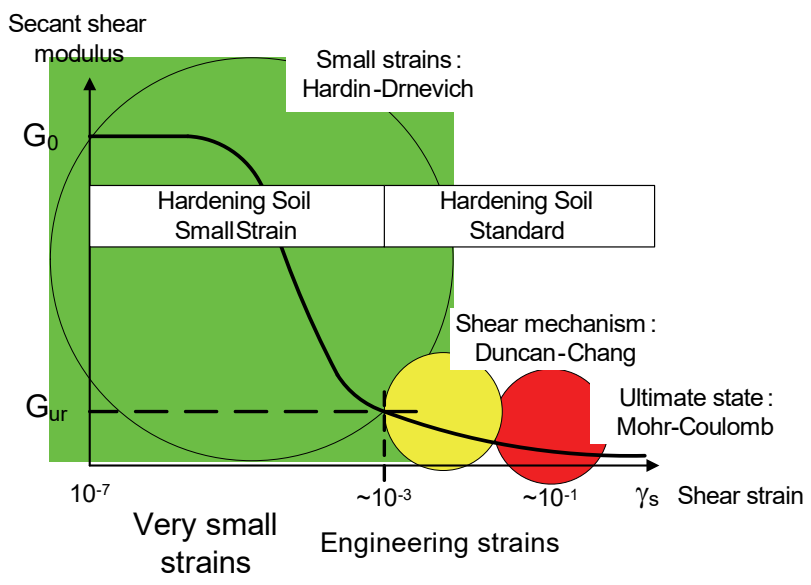

Fig. 6. Schematic representation of the Hardening-Soil model framework including a strong reduction of maximal shear stiffness that occurs with increasing amplitude of shear strain

An enhanced version of the HS-Standard, the Hardening Soil Small model (HS-SmallStrain) was formulated by Benz [5] in order to handle commonlyobserved phenomena of:

- strong stiffness variation with increasing shear strain amplitudes in the domain of small strains (Fig. 6),

- hysteretic, nonlinear elastic stress-strain relationship which is applicable in the range of small strains.

These features mean that the HS-SmallStrain is able to produce a more accurate and reliable approximation of displacements, which can be useful 
for dynamic applications or in the modeling of unloading-conditioned problems, e.g., deep excavations with retaining walls. The discussion on strong stiffness variation in low amplitudes of shear strains and small strain stiffness properties can be found in Dyvik and Madshus [8], Burland [7], Jardine et al. [10].

The implementation of the HS model in the ZSoil code (ZSO [1]) by Truty [17] allowed some improvements to be proposed with respect to Benz's version (Benz [5]), in order to simplify the numerical stress-strain integration scheme and enhance its robustness. The modifications were mostly related to the definition of the dilatancy law in the contractant domain (in this domain, the value of the mobilized friction angle $\phi_{m}$ is smaller than the critical state friction one $\phi_{c s}$ ) and description of the smooth (in the deviatoric plane) cap yield surface. For details, refer to Obrzud and Truty [11].

\subsection{BENDING MOMENTS}

A comparison of envelopes for characteristic bending moments $M_{k}$ derived from Method A and the 1st FE run for Method B are presented in Fig. 7. The form of envelopes is essentially the same. As expected, the first run of Method B gives larger bending moments than Method A due to greater wall rigidity. The bending moments derived from Method A simulation are $16 \%$ and $5.5 \%$ smaller than those ob- tained with the first run in Method B, inside and outside the excavation, respectively.

The design values of the bending moment $M_{d}$ were calculated based on the characteristic value obtained from the FE model using equation (1). These values were used to establish the minimal design crosssectional area of reinforcement $A_{\text {sd,min }}$ which was calculated using the procedure described in the Appendix. Table 2 shows that $A_{\text {sd,min }}$ computed for the bending moment derived from Method A and developed at the inner excavation side, is underestimated by about $17 \%$.

Figure 8 presents the critical distribution of bending moments along the retaining wall. The efforts are derived from the non-linear analysis of the retaining structure (2nd run of Method B), and the corresponding distributions of stress levels in the non-linear layers in beam elements for three selected cross sections. It can be noticed that the concrete tensile strength is fully mobilized for almost half of the wall cross sections for which the maximal bending moments are developed. The inward maximal bending moment, $M_{z}=676 \mathrm{kNm}$, is $30 \%$ smaller than that obtained for the "elastic" run with $E_{c}=34 \mathrm{GPa}$. This value is also smaller than $M_{z}=$ $757 \mathrm{kNm}$ obtained for $E_{c}=20 \mathrm{GPa}$ with Method A. It can be stated that the section considered is characterized by a strong local stiffness reduction in the wall member due to an excessive mobilization of concrete tensile resistance over the half of the cross section. Therefore, in the case of the 2nd run of Method B, we can expect larger wall deflections than those derived from the Method A simulation.

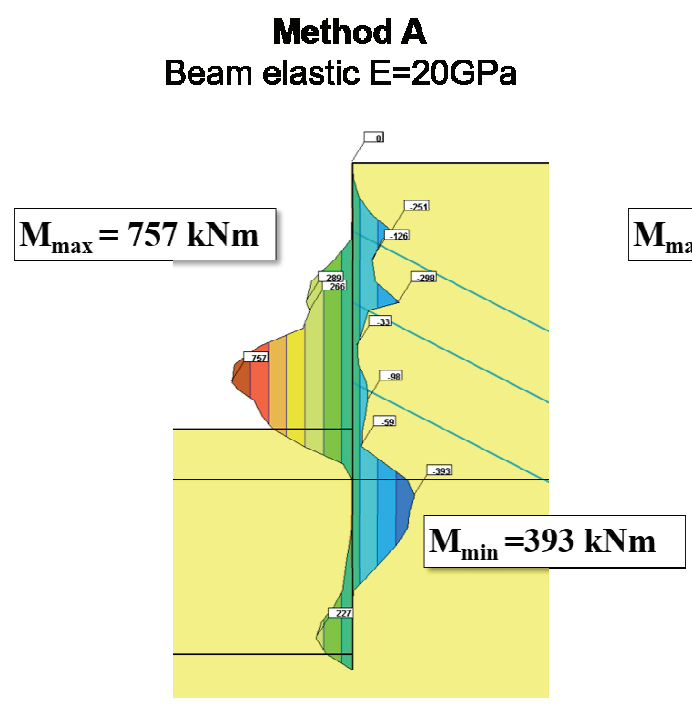

a)

\section{Method B (1st FE run) Beam elastic $E=34 \mathrm{GPa}$}

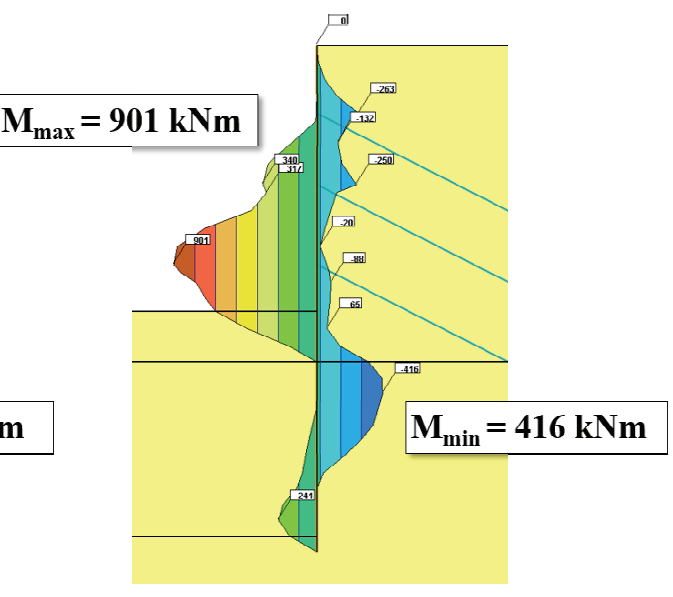

b)

Fig. 7. Comparison of the computed envelopes for bending moments in the retaining wall:

(a) Method A - elastic beam elements defined by $\mathrm{E}=20 \mathrm{GPa}$,

(b) 1st analysis for Method B assuming elastic beam defined by a nominal Young's modulus E $=34 \mathrm{GPa}$ 
Table 2. Minimal cross section area for reinforcement computed for the maximal design bending moment $M_{d}$

\begin{tabular}{|c|c|c|c|}
\hline \multirow{2}{*}{ Results } & Method A & Method B & $\begin{array}{c}\text { Relative } \\
\text { error* RE }\end{array}$ \\
\cline { 2 - 3 } & $E_{c}=20 \mathrm{GPa}$ & $E_{c}=34 \mathrm{GPa}$ & \\
\hline$M_{k}^{+}\left[\mathrm{kNm} / \mathrm{m}^{\prime}\right]$ & 757 & 901 & $-16 \%$ \\
\hline$M_{d}^{+}\left[\mathrm{kNm} / \mathrm{m}^{\prime}\right]$ & 1022 & 1216 & $-17 \%$ \\
\hline$A_{\mathrm{sd}, \text { min }}^{+}\left[\mathrm{mm}^{2}\right]$ & 3339 & 4016 & \\
\hline$M_{k}^{-}\left[\mathrm{kNm} / \mathrm{m}^{\prime}\right]$ & 393 & 416 & $-5.5 \%$ \\
\hline$M_{d}^{-}\left[\mathrm{kNm} / \mathrm{m}^{\prime}\right]$ & 531 & 562 & $-5.0 \%$ \\
\hline$A_{\mathrm{sd}, \text { min }}^{-}\left[\mathrm{mm}{ }^{2}\right]$ & 1690 & 1792 & \\
\hline \multicolumn{2}{|l|}{ Method B as the reference } &
\end{tabular}

Non-linear analysis, $A_{\mathrm{sd}, \min }$ obtained based on 1st run of method B

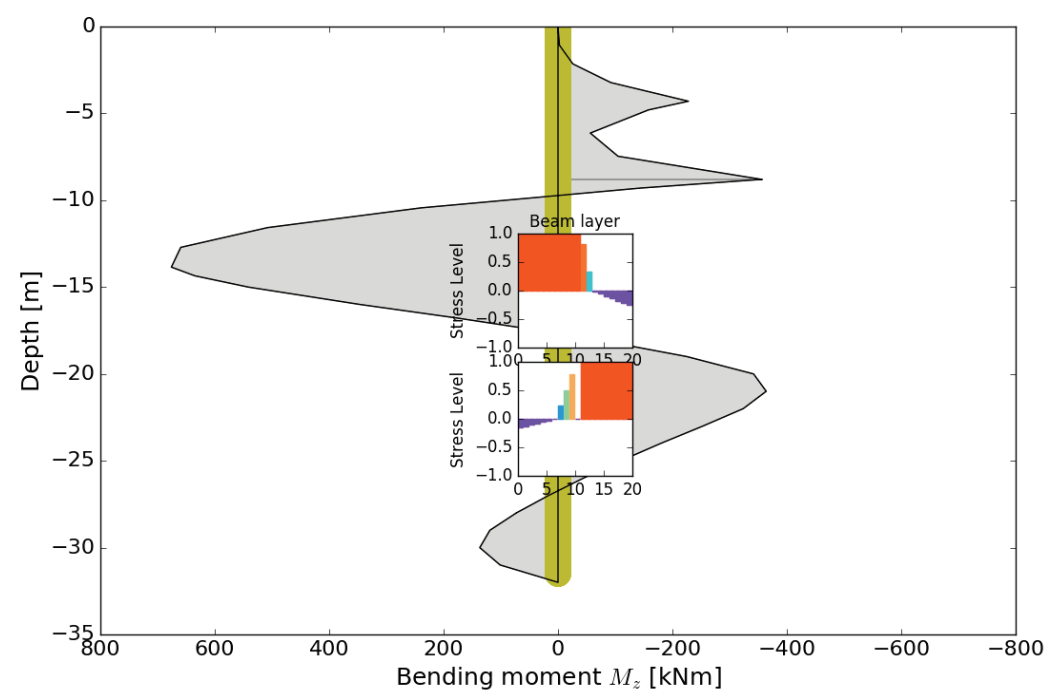

Fig. 8. Critical distribution of bending moments along the retaining wall derived from the non-linear analysis for the retaining structure (2nd run of Method B), and the corresponding distributions of stress levels in the non-linear layers in beam elements for two selected cross sections at the location of the maximal bending moments $M_{\max }$ from the inward and outward sides, respectively. Red bars stand for tension with 1.0 representing full strength mobilization, and blue bars meaning compression

\subsection{DISPLACEMENTS}

A comparison of maximal wall deflections and surface settlements derived from Method A and both Method B runs is presented in Fig. 9. The results are compared in Table 3. The distribution of displacement fields is essentially the same. As expected, the run of Method B gives smaller displacements than the simulation with the assumed crack of $E_{c}=20 \mathrm{GPa}$. As discussed before, a local stiffness reduction in the wall member obtained for the 2nd run of Method B, leads to larger displacements than those obtained with Method A. It means that in the case of non-linear structural simulation, the apparent rigidity of the wall is locally smaller than $20 \mathrm{GPa}$. However, the differences in computed displacements are not very high: $-14 \%$ for wall deflections and $10 \%$ for surface settlements.

Clearly, discrepancies between the results depend on the magnitude of mobilized deformations in soil, and it is difficult to clearly predict the trend since we deal with highly non-linear problems. The risk may arise and should be carefully examined when deep excavations take place next to adjacent buildings and services which were neglected in the above examples.

As discussed at the beginning of this section, experience shows that the internal forces obtained with Method A have occasionally been carelessly taken for 


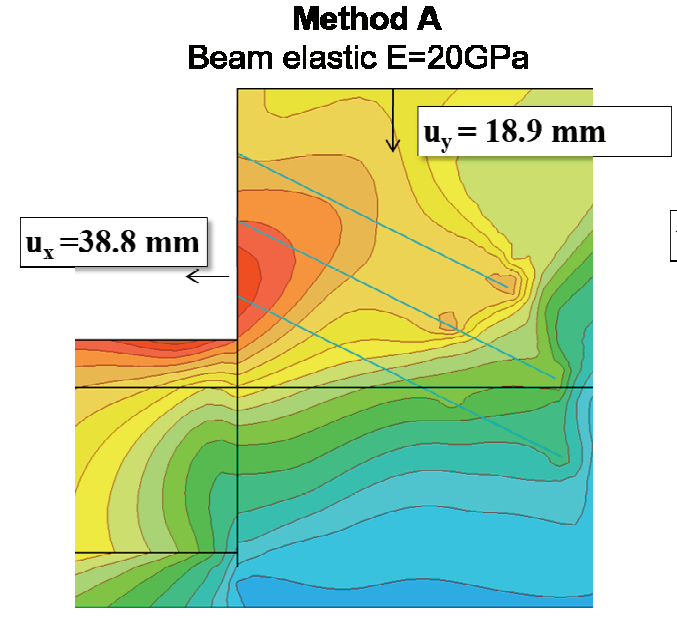

a)

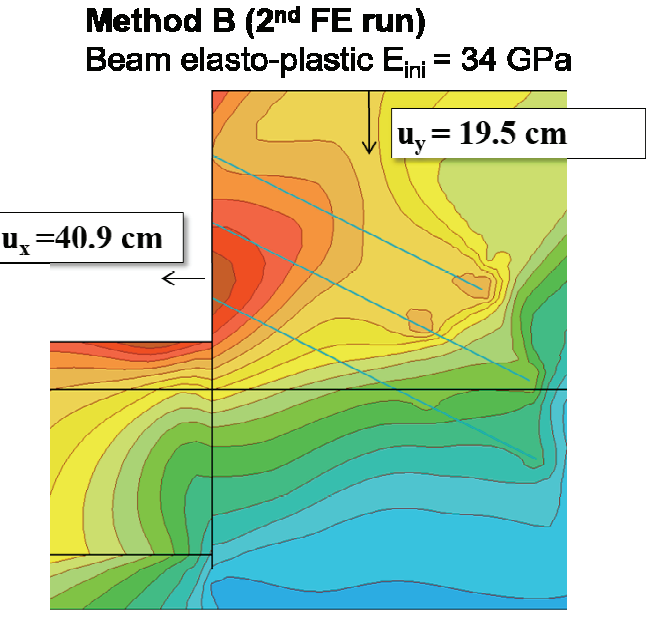

b)

Fig. 9. Comparison of displacement fields obtained with (a) the elastic beam model (Method A), and (b) elasto-plastic beam model (Method B)

Table 3. Comparison of maximal displacements obtained with Methods A and B

\begin{tabular}{|c|c|c|c|c|c|}
\hline \multirow[t]{3}{*}{ Results } & \multirow{3}{*}{$\begin{array}{c}\text { Method A } \\
E_{c}=20 \mathrm{GPa}\end{array}$} & \multicolumn{3}{|c|}{ Method B } & \multirow[t]{3}{*}{ Relative error* $\mathrm{RE}$} \\
\hline & & $E_{c}=34 \mathrm{GPa}$ & \multicolumn{2}{|c|}{$E_{c}^{i n i}=34 \mathrm{GPa}$} & \\
\hline & & & $\begin{array}{c}A_{\text {sd,min }} \text { based on } \\
\text { 1st run with } \\
\text { method B }\end{array}$ & $\begin{array}{l}A_{\text {sd,min }} \text { based on } \\
\text { single run with } \\
\text { method A }\end{array}$ & \\
\hline $\begin{array}{c}\text { Maximal horizontal } \\
\text { wall displacement } \\
u_{x}[\mathrm{~mm}]\end{array}$ & 38.8 & 35.2 & 40.9 & 42.0 & $-14 \%$ \\
\hline $\begin{array}{c}\text { Maximal settlement } \\
\text { behind the wall } u_{y} \\
{[\mathrm{~mm}]}\end{array}$ & 18.9 & 17.5 & 19.5 & 19.7 & $-10 \%$ \\
\hline
\end{tabular}

designing concrete members of the retaining structure. In such cases, the engineer should consider whether this strategy is consistent with respect to applicable design codes or standards.

\section{EFFECT OF ANALYSIS TYPE IN QUASI-IMPERMEABLE SOILS}

This section examines two approaches to modeling deep excavations in quasi-impermeable soils: the steady-state and consolidation analysis, respectively (Fig. 10). In the steady-state analysis, excess pore water pressure is dissipated at each excavation stage; this phenomenon can be observed in permeable soils, see Fig. 15. The steady-state analysis represents the ultimate pore pressure state which is obtained with the consolidation analysis. Consolidation is understood as a coupled, hydro-mechanical analysis. A non-linear consolidation algorithm simulates the transient behavior of the two-phase medium. Therefore, it is more suitable to analyze transient states during excavation in cohesive soils.

The main reason practitioners perform the steadystate analysis for modeling deep excavations in lowpermeable soils is because it is computationally less expensive (hydro-mechanical coupling in one direction). This analysis reduces the number of degrees of freedom by $30 \%$, resulting in a faster evaluation of first-order predictions. However, in the times of highperformance computers, such simplified modeling cannot be justified for 2D or medium-size 3D simulations up to approximately 300,000 degrees of freedom (DOFs); currently, ZSoil v2014 and v2016 allows simulations of more than $1,500,000$ to be performed. 


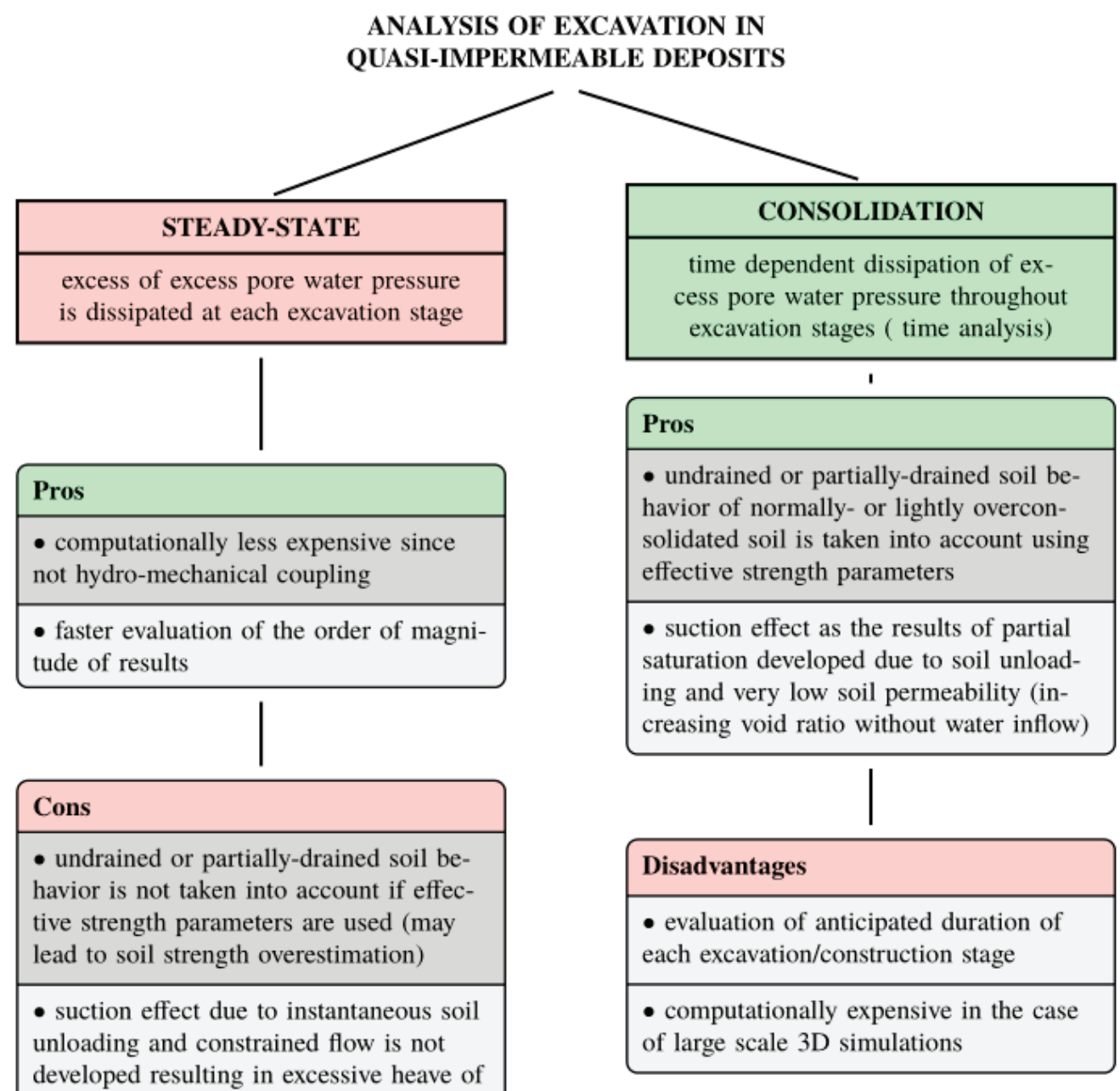

Fig. 10. Steady state vs consolidation for analyzing excavation problems in quasi-impermeable deposits

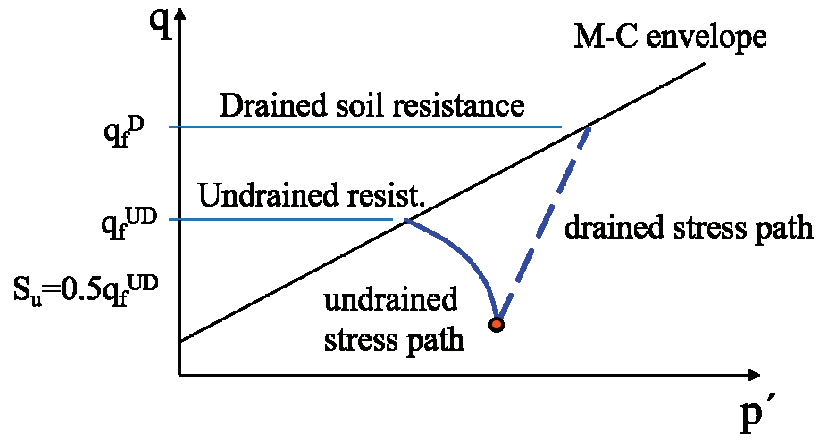

Fig. 11. Schematic representation of shear strength for normally consolidated material in drained and undrained conditions

Sometimes, applying the steady-state analysis and effective stress parameters which define advanced constitutive models can lead to an overestimation of soil resistance. It should not be forgotten that undrained or partially-drained conditions induce dif- ferent effective stress paths, especially in normally consolidated soils, as illustrated in Fig. 11. Moreover, suction effects due to instantaneous soil unloading and constrained water flow can be omitted in the analysis. It will be demonstrated that neglecting the suction effect yields in an excessive heave of the excavation bottom.

In conditions, unloading of saturated soils leads to the development of suction due to an increasing void ratio under constant water content. This results in partial soil saturation as the water inflow is constrained by low soil permeability. The transient consolidation algorithm can account for suction effects. Moreover, undrained or partially-drained behavior of normally- or lightly overconsolidated soil can be reasonably taken into account when using effective strength parameters. In such cases, the undrained shear strength depends on the current effective stress magnitude and the overconsolidation state (current position of the isotropic cap mechanism). 


\subsection{TWO-PHASE FORMULATION FOR PARTIALLY SATURATED MEDIA}

Appropriate modeling of soil-structure interaction problems using any advanced constitutive model for soil, and relying on effective stress parameters, requires considering a coupled analysis of deformation and pore water fluid flow. In most cases, part of the analyzed domain remains in the partially saturated zone, in which suction pressure exists and generates an apparent cohesion effect - suction. The zone below a free ground water table is typically considered as fully saturated. Therefore, the time scale effect may play an important role when analyzing typical geotechnical problems such as deep excavations, retaining walls, especially in poorly permeable deposits.

In the ZSoil finite element code, a consistent twophase formulation of a partially saturated medium is used according to the theory proposed by Aubry and Ozanam [4]. Due to a limited scope of this paper, we focus our attention only on the most important aspects of this formulation, limited here to the static cases.

In this theory, the overall equilibrium equation for the solid and fluid phases is written in the following form

$$
\begin{gathered}
\sigma_{i j, j}^{\mathrm{tot}}+\rho g b_{i}=0, \\
\rho=\rho_{\text {dry }}+n S \gamma_{F},
\end{gathered}
$$

with total stress components denoted by $\sigma_{i j}^{\text {tot }}$, gravity $g$, solid skeleton bulk density $\rho_{\text {dry }}$, water specific weight $\gamma_{F}$, porosity $n$ and current saturation ratio by $S$. The total stress obeys the effective stress principle after Bishop

$$
\sigma_{i j}^{\mathrm{tot}}=\sigma_{i j}+S p \delta_{i j}
$$

with $\delta_{i j}$ denoting Kronecker's symbol, $\sigma_{i j}$ effective stresses, and $p$ being the pore pressure.

The fluid flow continuity equation, including the effect of compressibility of the fluid and partial saturation, is expressed by the following equation

$$
S \dot{\varepsilon}_{k k}+v_{k, k}^{\mathrm{F}}=\left(n \frac{S}{K^{F}}+n \frac{\partial S}{\partial p}\right) \dot{p},
$$

with Darcy's velocity vector components denoted by $v_{k}^{F}$, the fluid bulk modulus $K^{F}$, and $n$ standing for porosity.

The extended Darcy's law, which accounts for seepage in the partially saturated zone (gas particles move with the same velocity as fluid ones) is the following

$$
v_{i}^{F}=k_{i j} k_{r}(S)\left(\frac{1}{\gamma_{F}} p_{, j}+b_{j}\right)
$$

where $\gamma_{F}$ denotes fluid unit weight, and the tensor of permeability for partially saturated medium is obtained by scaling the tensor for fully saturated medium $k_{i j}$ by a scalar valued function $k_{r}$ which depends on the actual, $S$, and residual saturation degree $S_{r}$ (after Irmay [9])

$$
k_{r}(S)=\left(\frac{S-S_{r}}{1-S_{r}}\right)^{3}
$$

The additional equations expressing relations $S(p)$ (a simplified van Genuchten's law (van Genuchten [19]) to model the soil water retention curve) are expressed as follows

$$
S=S(p)=\left\{\begin{array}{lll}
S_{r}+\frac{1-S_{r}}{\left[1+\left(\alpha \frac{p}{\gamma_{\mathrm{F}}}\right)^{2}\right]^{1 / 2}} & \text { if } & p<0 \\
1 & \text { if } & p \geq 0
\end{array}\right\}
$$

where $\alpha$ is a material constant which controls the rate of wetting or drying of a partially saturated medium. In other words, $1 / \alpha$ defines the height of the partiallysaturated zone so it can be taken as the inverse of the active capillary rise, whereas the residual saturation ratio can be defined as saturation that is attained after an initially saturated porous medium is allowed to drain by gravity to equilibrium conditions.

A graphical representation of equation (8) is presented in Fig. 12a.

The balance equation (3) and equation (6), written here in the strong form, can easily be converted to the weak form, and discretized by means of the standard Galerkin's procedure (Truty [15]). Further details concerning this issue and a comprehensive explanation of advanced stabilization techniques that are needed to handle quasi-undrained cases can be found in Truty [15], Truty and Zimmermann [16].

It is worth noting that suction pressures may generate a strong apparent cohesion effect. Considering Bishop's effective stress principle and van Genuchten's model, we can try to find a limit for the expression $S \cdot p$. For $S_{r}=0.0$ and $p \rightarrow-\infty$ this expression leads to the limit $S \cdot p \rightarrow \gamma_{F} / \alpha$ while for $S_{r}>0$ such a limit does not exist anymore. This artifact should be carefully considered in practical applications. 
a)

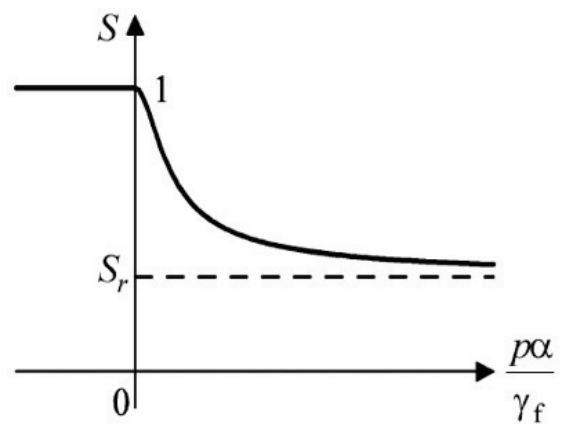

b)

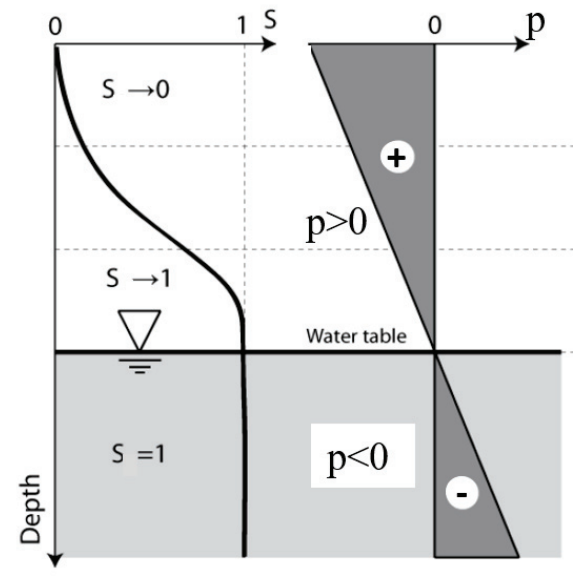

Fig. 12. Graphical representation of partially saturated medium:

(a) soil water retention curve by van Genuchten's model, and (b) schematic representation of the partially saturated zone above the ground water table

\subsection{NUMERICAL EXPERIMENT}

In order to demonstrate the effect of time-dependent analysis on soil displacements during excavations, non-cohesive soil was replaced with low-permeable, cohesive material in the previously analyzed model. The geometry of the excavation was taken from the first analysis and is presented in Fig. 13. The following modifications were introduced to render excavation in cohesive deposits:
- impermeable barrier was removed,

- zero pore pressures were imposed by applying seepage surface elements on the top of each of four excavation stages (see Fig. 14),

- effective friction angle for two clay layers was assumed $\phi^{\prime}=30^{\circ}$ and $\phi^{\prime}=32^{\circ}$, respectively, where as effective cohesion was assumed equal to $c^{\prime}=0 \mathrm{kPa}$,

- no dilatancy has been assumed for cohesive material following the discussion in Truty and Obrzud [18],

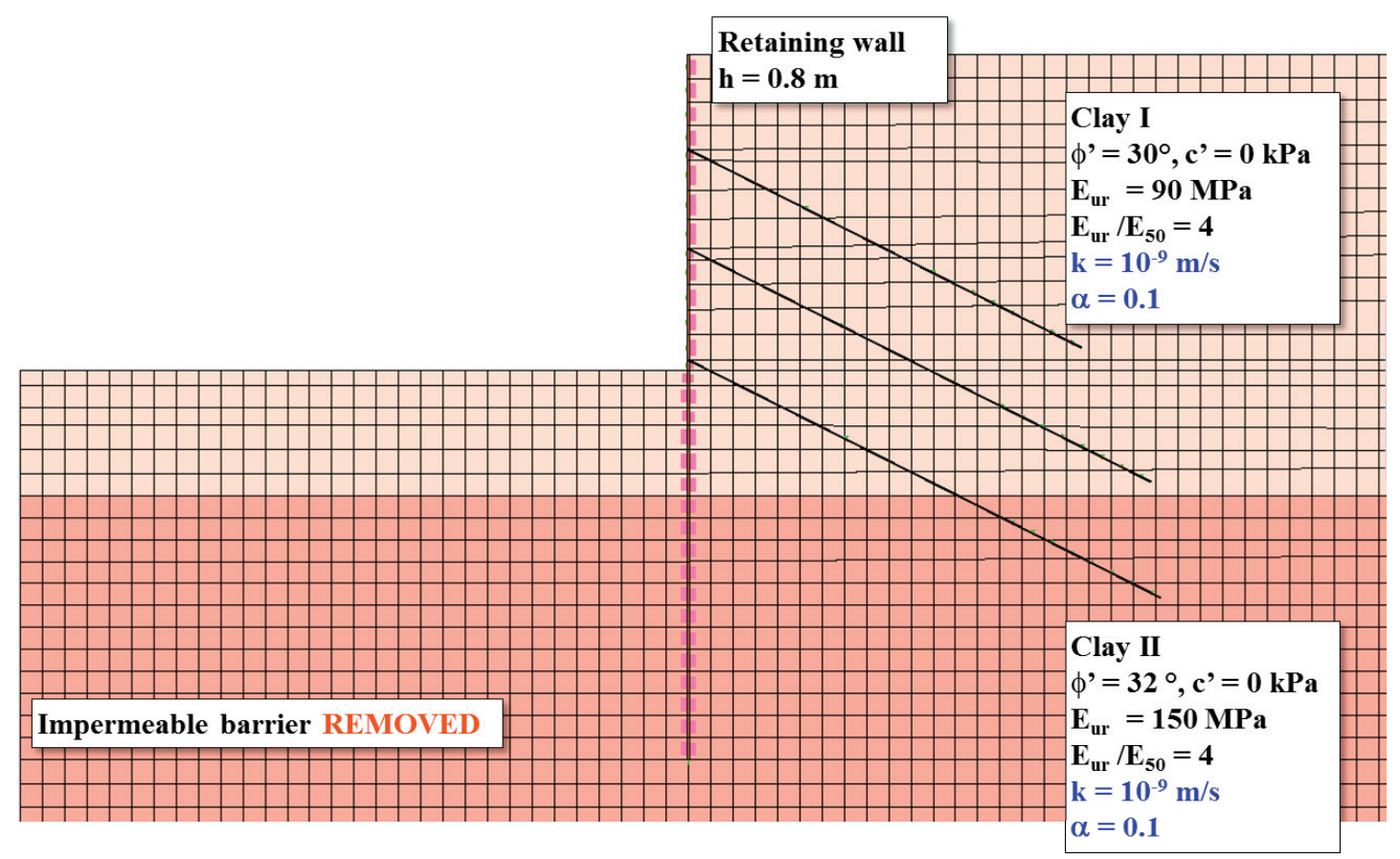

Fig. 13. Modification of the model presented in Fig. 4 for simulation of a hypothetical excavation in low permeable clays 


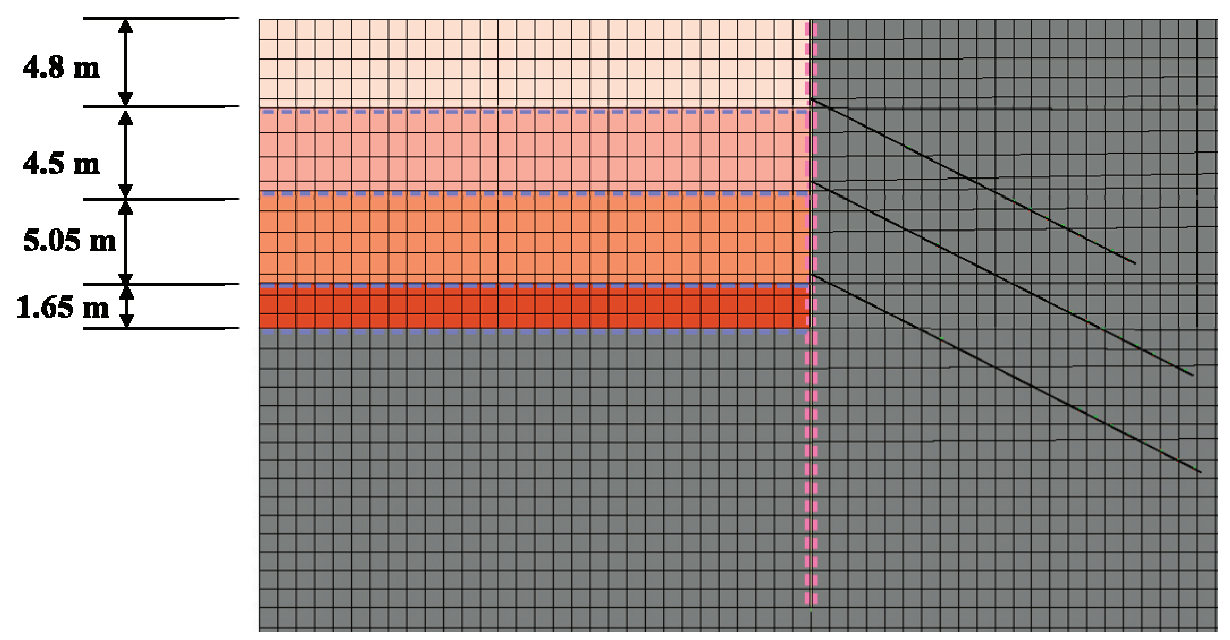

Fig. 14. Excavation stages considered in the FE simulation and the seepage surface elements applied at the bottom of each excavation level

\section{STEADY-STATE}

\section{CONSOLIDATION}

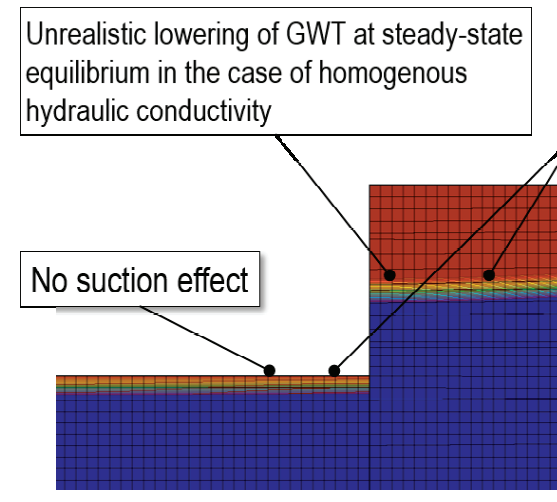

a)

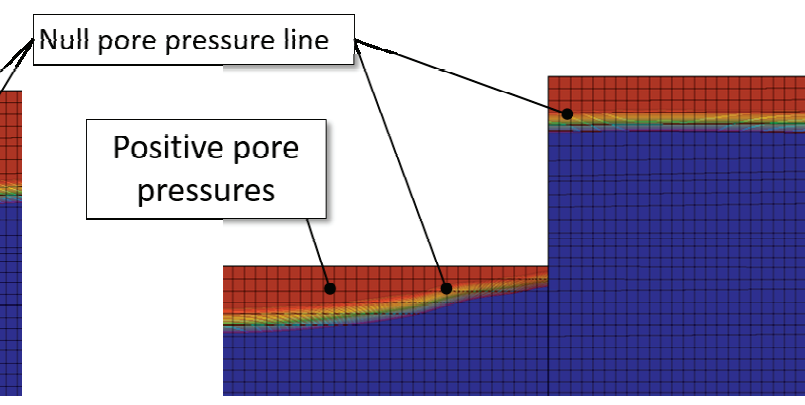

b)

Fig. 15. Pore pressure fields obtained for material with homogenous hydraulic conductivity:

(a) stady-state analysis: no suction effect due to imposed seepage at the excavation bottom;

(b) consolidation analysis: development of a positive pore pressure field due to partial saturation of soil

- low permeability clay was defined with the coefficient of hydraulic conductivity equal to $k=10^{-9} \mathrm{~m} / \mathrm{s}$, and a low saturation constant ${ }^{2}$ value: $\alpha=0.1$.

Similarly to the previously analyzed model, the excavation was carried out in four stages. The timedependent analysis was discretized so that each stage lasted 30 days, during which a partial pore pressure dissipation could take place. In order to account for the 3D effect of excavation in an out-of-plane direction, a gradual unloading was applied for the first 5 days. After the first 20 days, pre-stressed anchors were installed.

Finally, in order to consider two equivalent hydrological systems when comparing steady-state and consolidation analyzes, a high permeable soil layer was introduced at the ground water level for the steady-state analysis model. This layer ensures a constant ground

\footnotetext{
${ }^{2}$ Parameter $\alpha$ can be taken as the inverse of the capillary rise.
}

water level behind the wall similar to that computed with the consolidation analysis (Fig. 15b). This problem may occur for a boundary value problem where the subsoil is characterized by homogenous hydraulic conductivity, as demonstrated in Fig. 15a.

In the first analysis, the wall was considered elastic and defined with $E_{c}=34 \mathrm{GPa}$. A comparison of computed results can be started by showing the suction effect at the bottom of the excavation 30 days after the excavation stage, Fig. 16 (about $30 \%$ of consolidation). The unsaturated zone which is developed below the excavation reaches about $4.0 \mathrm{~m}$. Knowing that the total stresses $\sigma_{\text {tot }}$ at the excavation surface equal zero, the effective stresses $\sigma^{\prime}$ are equal to the suction $S \cdot p$ which is about $20 \mathrm{kPa}$ in this case. The non-zero effective stress results in higher soil resistance in the passive side of the wall than that computed for steady-state approach. Soil resistance for the steady- 


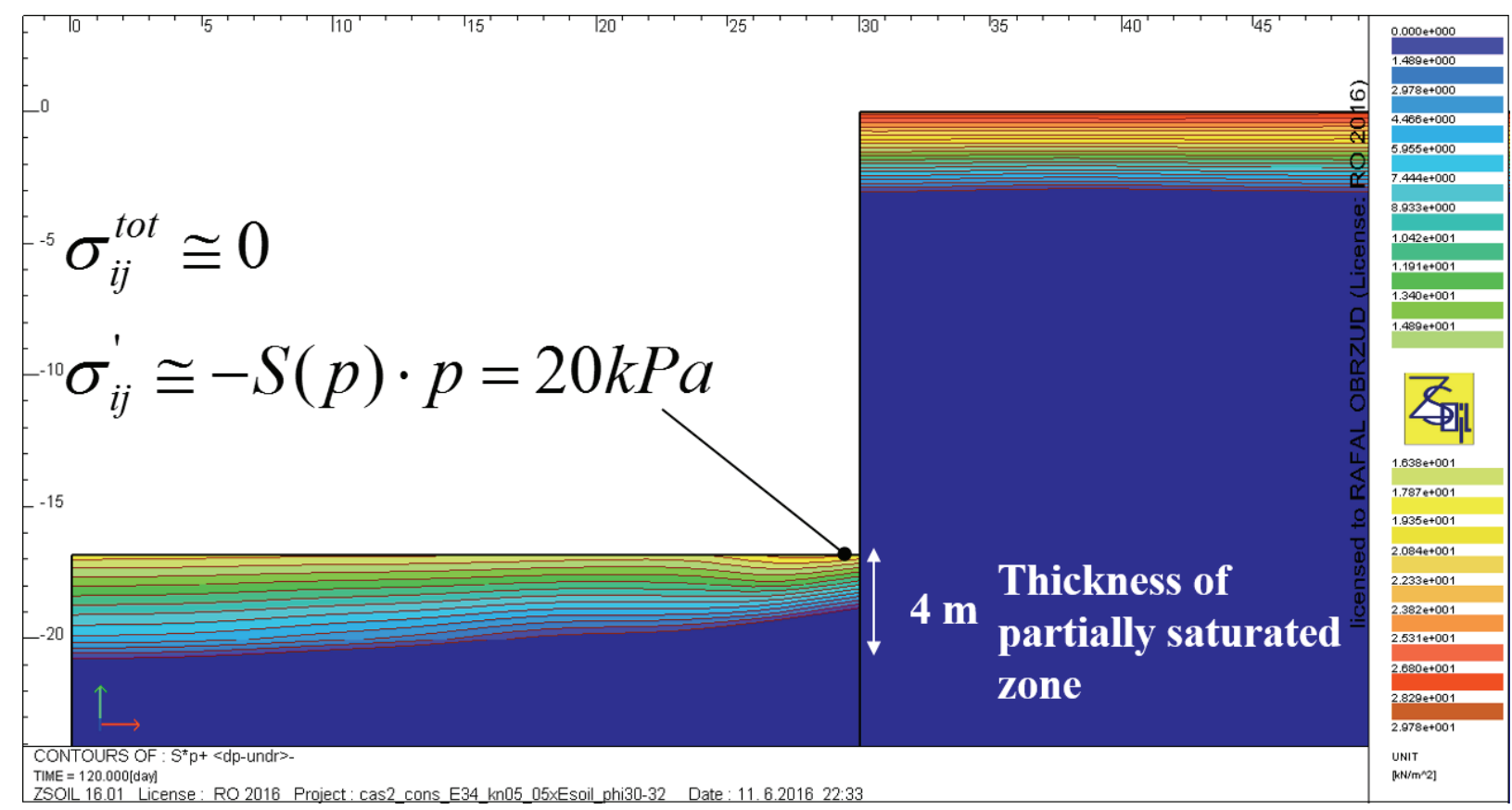

Fig. 16. Suction effect at the bottom of the excavation 30 days after excavation stage

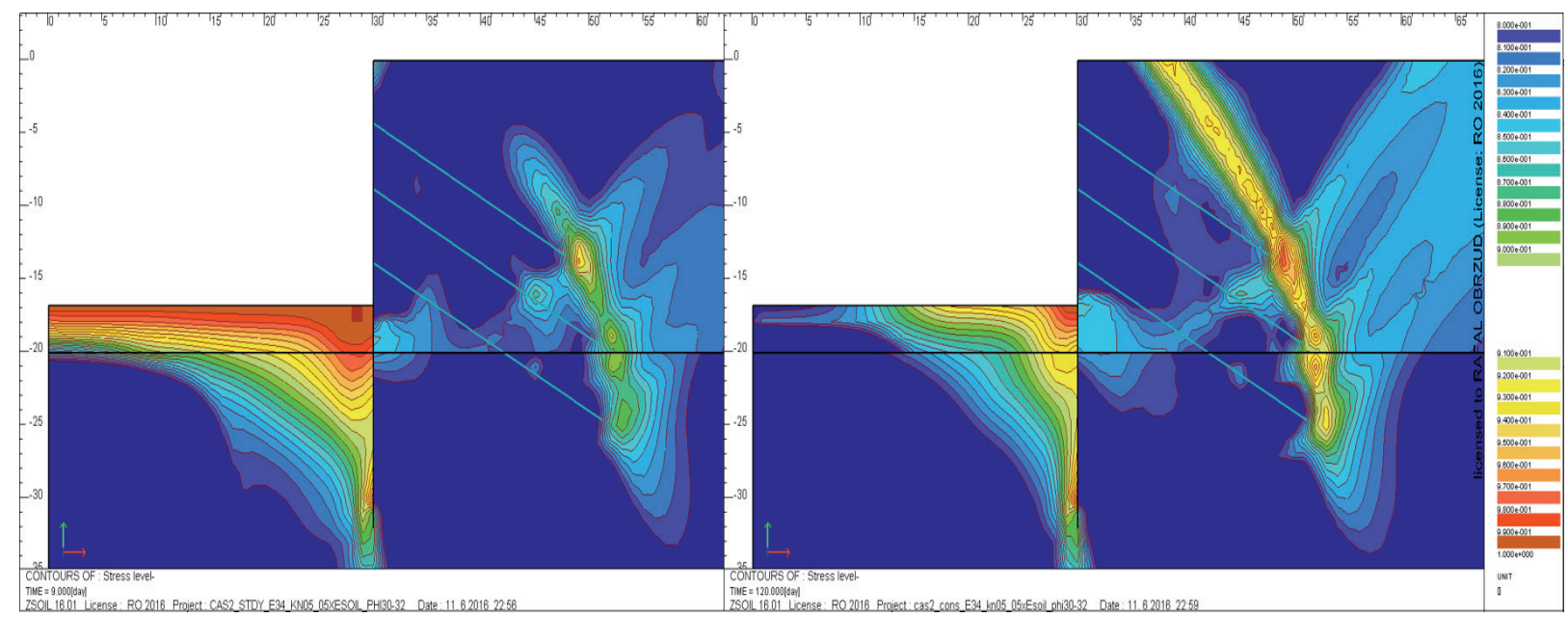

a)

b)

Fig. 17. Representation of stress level between 0.8 and 1.0 (marked in red) for (a) steady-state, and (b) consolidation analysis. Stress level 1.0 representing full mobilization of soil strength

state analysis is conditioned by seepage-imposed null pore pressures inducing $\sigma^{\prime}=0$. Strength mobilization for both analyzes is represented by means of stress level maps in Fig. 17.

As a result of lower passive soil resistance, steadystate computation gives significantly larger displacements. Settlements in the active zone are about $100 \%$ higher than those computed with a consolidation driver. Wall deflections and excavation bottom heave are also higher, about 70\%, as shown in Fig. 18.
It can also be shown that the maximal inward bending moments are $52 \%$ higher for the steady-state approach (refer to Fig. 19). At this point, it can be concluded that for the example considered, steadystate computing leads to a very conservative design in terms of required minimal design cross sectional area of reinforcement $A_{\mathrm{sd}, \min }$.

In the final step of the experiment, a comparison of two SLS checks was carried out. In the first simulation, the analysis is carried out for the elastic beam 
Steady - state

Beam elastic $\mathrm{E}=34 \mathrm{GPa}$

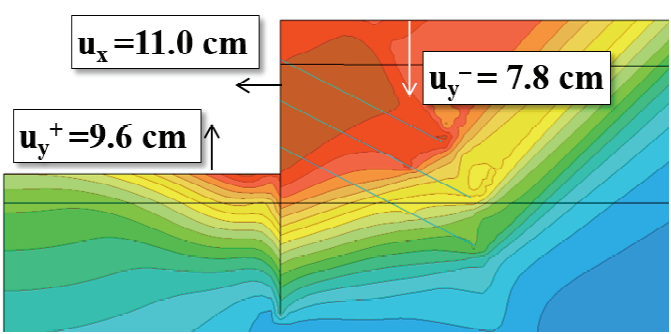

\section{Consolidation}

Beam elastic $\mathrm{E}=34 \mathrm{GPa}$
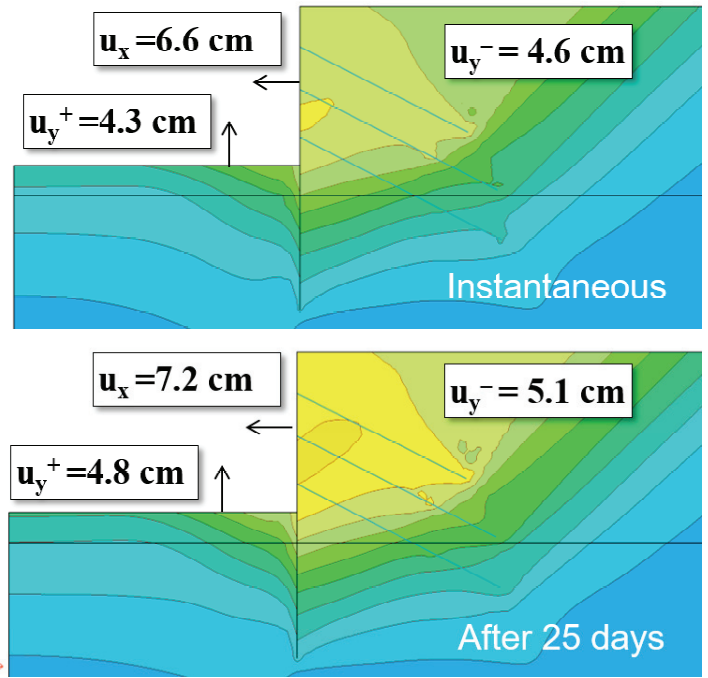

b)

a)

Fig. 18. Comparison of maximal displacements derived from: (a) steady-state analysis, and (b) consolidation analysis, for the elastic wall

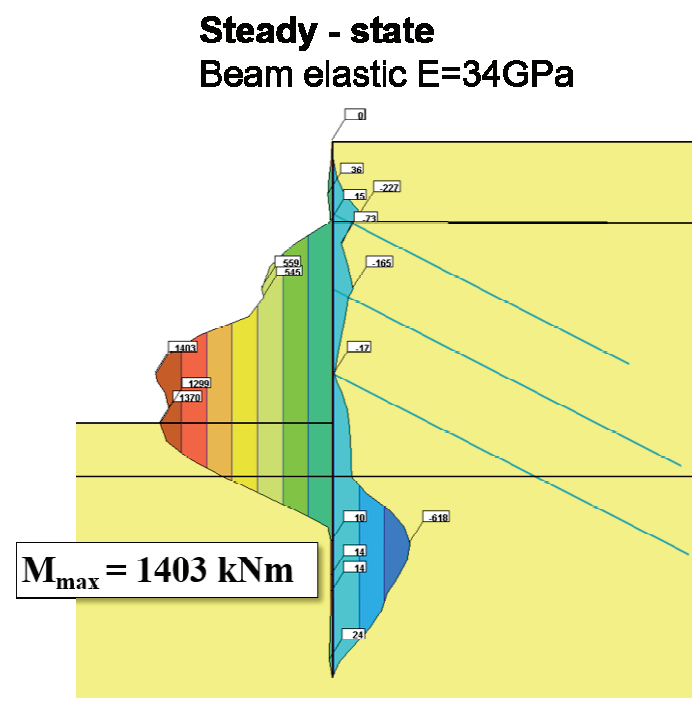

a)

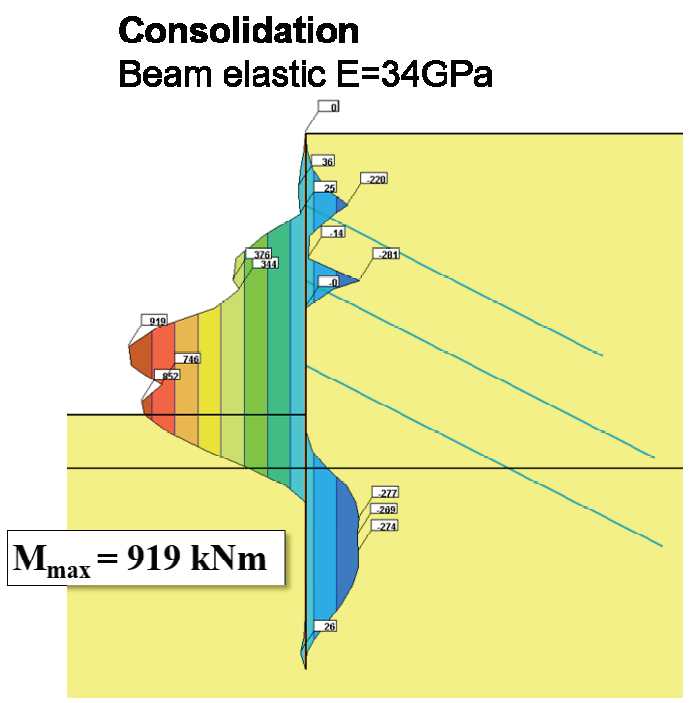

b)

Fig. 19. Comparison of a characteristic bending moment envelopes from: (a) the steady-state analysis, and (b) consolidation analysis, for the elastic wall

with $E_{c}=20 \mathrm{GPa}$ and the steady-state approach. The second simulation combines the consolidation with the non-linear structural analysis. Hence, this simulation can be viewed as the most genuine SLS verification for the boundary value problem considered and assumed hypotheses.

The obtained results are presented in Fig. 20 and are compared in Table 4 . It can be noticed that the combination of the steady-state analysis and Method A gives very conservative predictions in terms of displacements. The maximal settlement and wall deflection are about $55 \%$ higher than those computed with the "actual" SLS check. Moreover, the "actual" SLS check gives a more istic prediction of excavation bottom heave due to the suction effect that occurs in unloaded soil. 
Steady - state + Method A

Beam elastic $\mathrm{E}=20 \mathrm{GPa}$

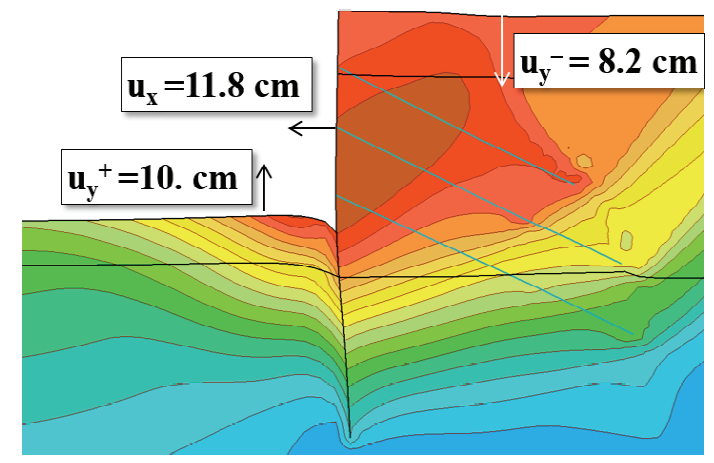

a)
Consolidation + Method B Beam elasto-plastic $E=34 \mathrm{GPa}$ $A_{d}=A_{d}\left(M_{\max }\right)$

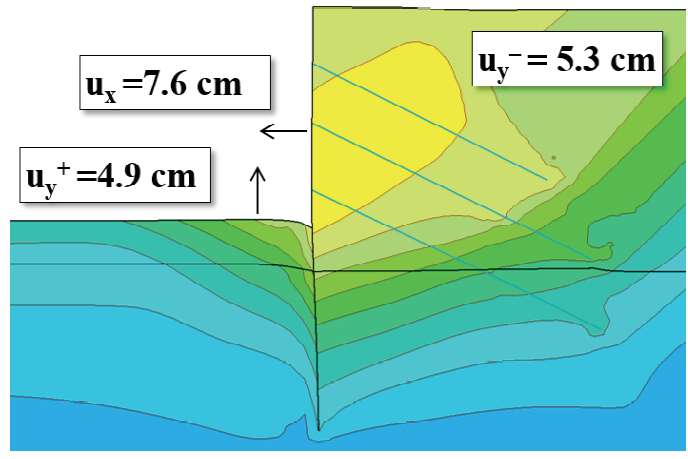

b)

Fig. 20. Comparison of displacements obtained with two SLS checks:

(a) steady-state analysis with elastic beam $\left(E_{c}=20 \mathrm{GPa}\right)$,

and (b) "genuine" SLS verification combining consolidation and non-linear structural analysis

Table 4. Comparison of maximal displacements obtained for two analysis types

\begin{tabular}{|c|c|c|c|c|}
\hline Results & $\begin{array}{c}\text { Steady-state } \\
\text { analysis combined } \\
\text { with Method A }\end{array}$ & $\begin{array}{c}\text { Consolidation } \\
\text { type analysis } \\
\text { combined } \\
\text { with Method A }\end{array}$ & $\begin{array}{c}\text { Consolidation } \\
\text { type analysis } \\
\text { combined } \\
\text { with Method B }\end{array}$ & $\begin{array}{c}\text { Relative } \\
\text { error* RE }\end{array}$ \\
\hline$E_{c}=20 \mathrm{GPa}$ & $E_{c}=20 \mathrm{GPa}$ & $\begin{array}{c}E_{c}^{\mathrm{ini}}=34 \mathrm{GPa} \\
A_{d}=A_{d}\left(M_{\max }\right)\end{array}$ & 76 & $55 \%$ \\
\hline $\begin{array}{c}\text { Maximal horizontal } \\
\text { wall displacement } u_{x} \\
{[\mathrm{~mm}]}\end{array}$ & 118 & 75 & 53 & $55 \%$ \\
\hline $\begin{array}{c}\text { Maximal settlement } \\
\text { behind the wall } \\
u_{y}[\mathrm{~mm}]\end{array}$ & 82 & 53 & 49 & $104 \%$ \\
\hline $\begin{array}{c}\text { Maximal heave at } \\
\text { excavation bottom } \\
u_{y}[\mathrm{~mm}]\end{array}$ & 100 & 49 & & \\
\hline \multirow{2}{*}{ Steady-state analysis with respect to consolidation one. } & \\
\hline
\end{tabular}

\section{CONCLUSIONS}

In the first part of this paper, two approaches to SLS verification for the deep excavation boundary value problem are examined. In the first approach (Method A), the diaphragm wall is modeled with the Hookean material, assuming the globally reduced stiffness equals $20 \mathrm{GPa}$ due to possible concrete cracking. The second approach (Method B) is divided into two stages. In the first FE run, the wall is modeled with the highest nominal Young's modulus equal to $34 \mathrm{GPa}$. This modulus makes it possible to find design bending moments which are used to compute the minimal design cross-section reinforcement for the retaining structure. The computed reinforcement is then used in a non-linear structural analysis which is viewed as the "actual" SLS verification. In method B, the stiffness of the wall is reduced locally and not equally depending on the magnitude of bending moments.

In the example presented, the global reduction method gives a smaller magnitude of displacement of about $10-15 \%$ compared to the "actual" SLS verification. However, we should be aware that the order of magnitude for discrepancies can vary from one case to 
another since the nature of geotechnical problems is highly non-linear.

It is also discussed that the internal forces obtained with Method A should be carefully taken when designing reinforcement for concrete members in the retaining wall. In such a case, an engineer should consider whether this approach is consistent with applicable design codes or standards, and - most importantly - should take into account the associated risk.

In the second part, the same boundary value problem is re-analyzed assuming that the excavation takes place in low-permeable, cohesive material. In the numerical experiment, the "actual" SLS check relying on time-dependent consolidation analysis is compared with the steady-state analysis which should be considered as a simplification in the light of constrained water exchange during relatively rapid excavation.

It has been illustrated with the aid of the transient analysis that the suction effects play an important role in estimating soil deformations for deep excavation problems. The unsaturated zone which is developed below the excavation bottom can be thick. This suction which is associated with the partially-saturated soil, induces higher strength resistance in the passive zone due to nonzero effective stress at the bottom of excavation. The displacements obtained with the transient analysis are significantly smaller than those derived from steady-state analysis. Is the steady-state analysis in this case appropriate in the light of optimal excavation design?

\section{APPENDIX: CROSS SECTIONAL AREA OF REINFORCEMENT}

In this paper, the minimal cross sectional area of reinforcement was computed assuming rectangular stress distribution model (Fig. 21) and applying the following procedure:
The initial diameter $\phi=30 \mathrm{~mm}$ for rebars was assumed.

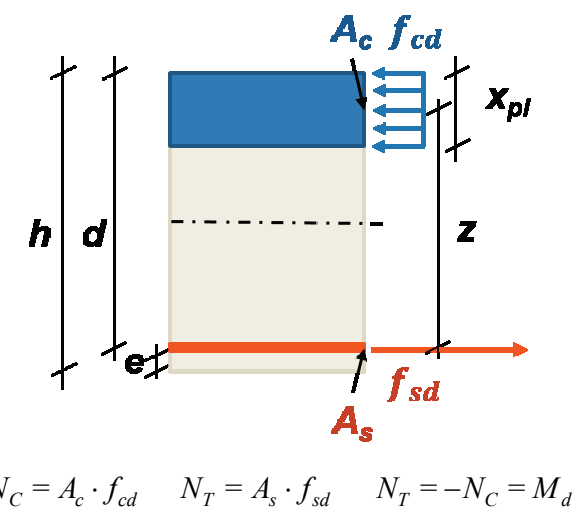

Fig. 21. Rectangular stress distribution model adopted for calculating reinforcement of the cross section for the wall members

\section{REFERENCES}

[1] ZSoil manual, Elmepress and Zace Services Limited, Lausanne, Switzerland 2014.

[2] EN 1992-1-1 (2002): Eurocode 0: Basis of structural design. The European Union Per Regulation 305/2011, Directive 98/34/EC, Directive 2004/18/EC.

[3] EN 1992-1-1 (2004): Eurocode 2: Design of concrete structures - Part 1-1: General rules and rules for buildings. The European Union Per Regulation 305/2011, Directive 98/34/EC, Directive 2004/18/EC.

[4] Aubry D., OzAnam O., Free-surface tracking through nonsaturated models, [in:] Swoboda (ed.), Numerical Methods in Geomechanics, Balkema, Innsbruck, 1988, 757-763.

[5] BENZ T., Small-strain stiffness of soils and its numerical consequences, $\mathrm{PhD}$ thesis, University of Stuttgart 2006.

[6] Burland J.B., Simpson B., St John H.D., Movements around excavations in London Clay, Proc. 7th ECSMFE, Brighton 1979, Vol. 1, 13-29.

[7] BuRLAND J.B., Small is beautiful - the stiffness of soils at small strains, 9th Bjerrum Memorial Lecture, Canadian Geotechnical Journal, 1989, Vol. 26, 499-516.

[8] DYVIK R., MaDSHUS C., Laboratory measurements of Gmax using bender elements, Norwegian Geotechnical Institute, Oslo, Norway, 1985, Publication No. 161, 186-196.

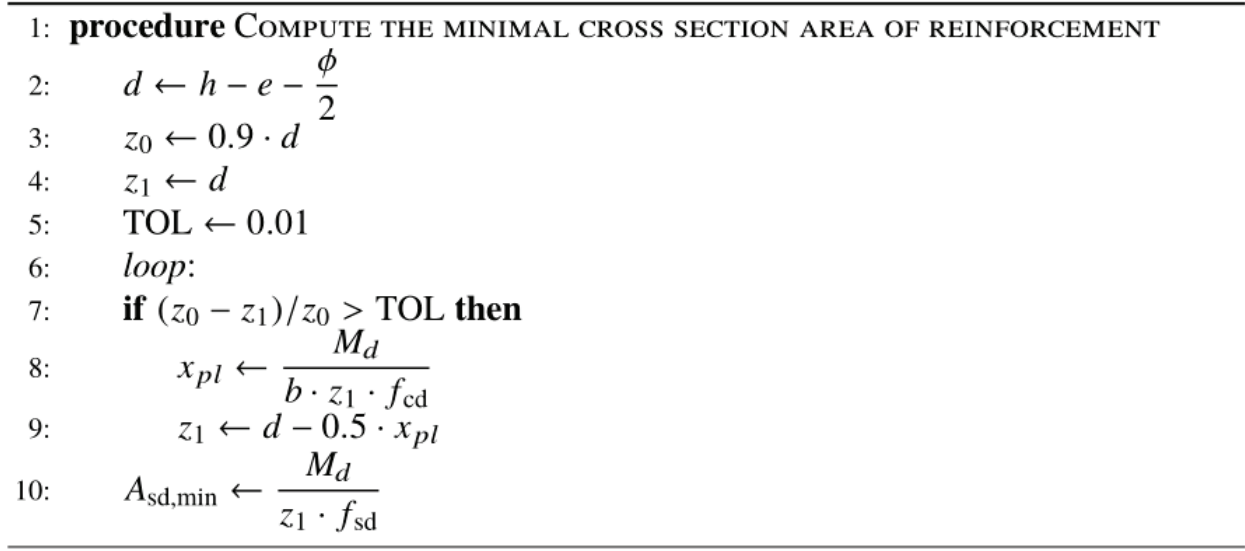


[9] IRMAY S., On the hydraulic conductivity of unsaturated soils, Trans. Am. Geophys. Union, 1956, 35, 463-468.

[10] Jardine R.J., PotTs D.M., Fourie A.B., Bourland J.B., Studies of the influence of non-linear stress-strain characteristics in soi -structure interaction, Géotechnique, 1986, Vol. 36, No. 3, 377-396.

[11] OBRZUd R., TRUTY A., The hardening soil model- a practical guidebook, edition 2016, Technical Report Z Soil. PC 100701, Zace Services Ltd. 2011.

[12] Schanz T., Zur Modellierung des mechanischen Verhaltens von Reinbungsmaterialien, Mitt. Inst. für Geotechnik 45. Universitat Stuttgart 1998.

[13] Schanz T., Vermeer P., Bonier P., Formulation and verification of the Hardening Soil Model, [in:] Beyond 2000 in Computational Geotechnics, Balkema, Rotterdam 1999.

[14] SCHWEIGER H.F., Benchmarking in Geotechnics, CGGIR006-2002, Graz University of Technology, Austria 2002.
[15] TRUTY A., On certain classes of mixed and stabilized mixed finite element formulations for single and two-phase geomaterials, Zeszyty Naukowe Politechniki Krakowskiej, Seria Inżynieria Środowiska 48, Kraków 2002.

[16] TRUTY A., ZimmermanN T., Stabilized mixed finite element formulations for materially nonlinear partially saturated two-phase media, Computer Methods in Applied Mechanics and Engineering, 2006, 195, 1517-1546.

[17] TRUTY A., Hardening Soil model with small strain stiffness, Technical Report Z Soil.PC 080901, Zace Services Ltd 2009.

[18] TRUTY A., OBRZUD R.F., Improved formulation of the Hardening Soil model in the context of modelling the undrained behaviour of cohesive soils, Studia Geotechnica et Mechanica, 2015, Vol. 37, No. 2.

[19] VAN GENUCHTEN M.T., A closed form equation for predicting the hydraulic conductivity of unsaturated soils, Soil Sciences Am. Soc., 1980, 44, 892-898. 